\title{
The bones of contention
}

\section{The skeleton may provide more than just structural support. Alla Katsnelson investigates the rise of} bone as a metabolic regulator.

$\Lambda$ t first, Patricia Ducy was not particularly fond of the mice she found herself working with in 1994. One of the first strains genetically engineered to lack a bone-related protein - osteocalcin their cages reeked of urine. And when Ducy dissected the mice, their bellies overflowed with fat. "They are disgusting," Ducy remembers telling her supervisor. "They pee so much; I hate them!"

Ducy was a postdoc at the University of Texas MD Anderson Cancer Center in Houston. Working with junior professor Gerard Karsenty, she was using the mice to test the idea that osteocalcin helped regulate the way calcium phosphate is deposited onto bone cells. Although the fat, smelly mice grew slightly stronger and thicker bones than ordinary mice, osteocalcin didn't seem to have any effect on mineralization. Its function remained a mystery. However, for Karsenty, who had originally trained in Paris as an endocrinologist, there was something familiar about the unfortunate rodents' symptoms: they seemed to be diabetic.

Maybe, he thought, the osteocalcindeficient mice indicated a direct link between the skeleton and energy metabolism, which goes askew in diabetes. The connection made sense to Karsenty. The body's maintenance of bone is a highly dynamic process orchestrated by two types of cell. Osteoblasts form and shape bone tissue, and osteoclasts break it down. Karsenty reasoned that this energy-intensive back-andforth process of remodelling might be coupled by necessity to metabolism. Adult humans who don't eat - such as those with anorexia - lose bone mass, and obese people have beefy bones. Obesity even seems to protect some women from osteoporosis.

The idea was radical. Most scientists who study bone believed the role of the skeleton was limited to support and mineral exchange, and the possibility that it could interact with other organ systems was unheard of. But over the past 15 years, Karsenty and Ducy, who "The best way
to address
scrutiny is if
your neighbour
gets the same
results as you."

implications. "Gerard has really been one of the most creative thinkers in the bone field for many years," says Sundeep Khosla, an endocrinologist at the Mayo Clinic in Rochester, Minnesota. Still, Karsenty is not without detractors, who say he has overstated the case for a bone-metabolism connection. And because almost no one in the field has worked directly on the idea, "the question people have is validation in another lab", says Khosla.

That is beginning to change. In a study published last month ${ }^{1}$, bone researchers at Johns Hopkins University in Baltimore, Maryland, largely stumbled on findings that affirm bone's role in metabolism. It has taken a long time, however, to put the pieces together.

\section{The missing link}

Karsenty put his osteocalcindeficient mice aside for a time in the late 1990s and plunged into his new hypothesis using a protein with a well-established metabolic role: leptin. The gene for leptin which is secreted by fat cells and suppresses appetite - had been cloned, and by then, a mouse in which the leptin gene was deleted (a knockout) was commercially available. More intriguing for Karsenty, leptin had been found only in animals with bony skeletons, suggesting that it evolved with bone. Perhaps, he thought, the hormone also serves as a 'molecular link' connecting energy metabolism to bone's growth and decay.

When the leptin-knockout mice arrived in late 1998, it was clear by a quick glance at an $\mathrm{X}$-ray that their bones were denser than those of wild-type mice. By then, Karsenty and his group had moved to Baylor College of Medicine, also in Houston, and in 1999 Karsenty brought in another postdoc, endocrinologist Shu Takeda. Initially, Takeda says, he didn't believe the idea would pan out, but the findings supported are now married and run collaborating labs at Columbia University in New York, have gradually shifted the bulk of their work towards testing the hypothesis, which could have clinical
Karsenty's vision. The knockout mice had a 40-50\% increase in bone mass compared with wild-type mice, suggesting that leptin blocks bone growth. But leptin didn't seem to be
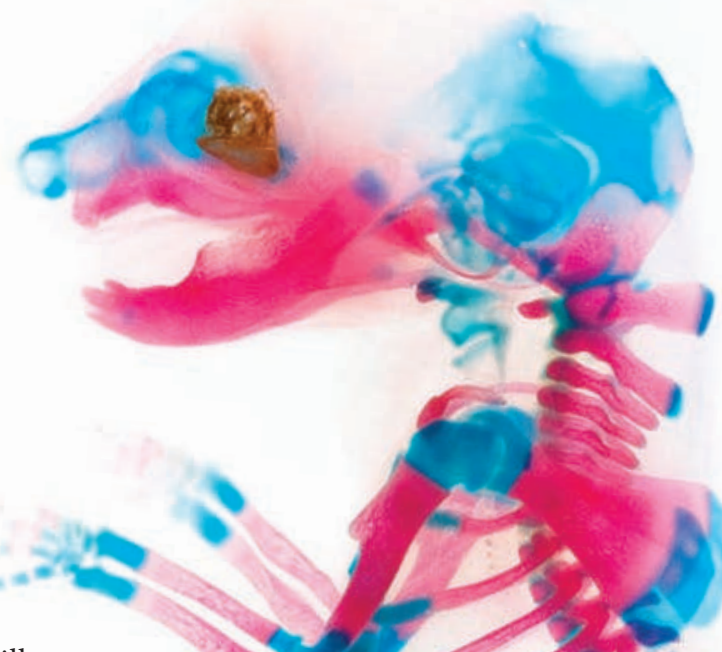

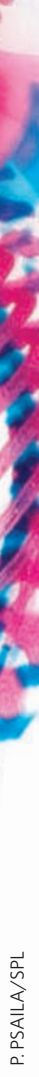

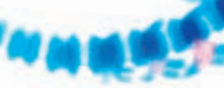

Developing skeleton of a mouse embryo. Red denotes bone and blue denotes cartilage.

signalling directly to bone cells. The researchers had to infuse it into the brain to return the knockouts' bones to normal ${ }^{2}$.

Karsenty's lab spent the next decade filling in details of the signalling pathway that connected leptin, osteocalcin and bone (see graphic), piecing the puzzle together largely by knocking out different genes. Most homeostatic processes are regulated by the brain, and this one was no exception. Leptin exerts its brake-pedal effect on bone growth by inhibiting synthesis of the neurotransmitter serotonin in the brainstem. This in turn dials down bone building - which is regulated by the sympathetic nervous system, a neuronal pathway that controls many unconscious bodily functions ${ }^{3}$. But the endocrine system works by feedback loops. If a hormone released from fat cells can affect bone metabolism, the scientists reasoned, bone must in turn regulate energy 
metabolism. And when they went back to their osteocalcin knockouts they confirmed that the mice were glucose intolerant, insulin resistant, and had fewer insulin-producing $\beta$-cells than normal mice - they were diabetic. Karsenty concluded that an activated form of osteocalcin acts as an energy-regulating hormone ${ }^{4}$.

The idea still hadn't gained much traction when Thomas Clemens, a bone biologist at Johns Hopkins University, came to study it. In 2006, one of his graduate students, Keertik Fulzele, became interested in the conflicting medical literature on whether or not diabetic patients, who have poor insulin-mediated control of glucose, are prone to osteoporosis. Fulzele suggested knocking out the insulin receptor specifically in osteoblasts. "I did everything I possibly could to discourage him," says Clemens, who was convinced that they'd see an uninformative effect, but Fulzele insisted.

Like the osteocalcin and leptin knockouts, the insulin-receptor-knockout mice were fat and insulin-resistant, but unlike the other two mutants, they had low bone mass. It was puzzling, Fulzele recalls. "We spent about six or eight months arguing about it and doing all sorts of controls." Just then, the Karsenty lab's 2007 paper ${ }^{4}$ was published, and for both Clemens and Fulzele, the light dawned. Bone and metabolism were connected.

Coincidentally, Karsenty and his team had created precisely the same knockout, and emerged with a similar story. Eating promotes insulin release, which activates bone remodelling. Bone formation by osteoblasts produces more osteocalcin, and bone resorption (or break down) by osteoclasts activates the hormone, releasing it from bone into the bloodstream. Osteocalcin in turn acts on pancreatic $\beta$-cells to boost insulin production and hence glucose uptake in the body. The two studies ${ }^{1,5}$ were published together in Cell this July.

Karsenty and Clemens suggest that this bone pathway may facilitate glucose absorption on a different time frame from the well-established insulin pathway, by which a jump in glucose levels spurs the quick release of insulin. That direct pathway would provide a rapid-response system for glucose uptake, whereas the bone pathway might oversee the longer-term equilibrium. Findings from the osteoblast insulin-receptor knockouts ${ }^{1,5}$, "sort of complete the loop that insulin is really one of the feedbacks on the bone-remodelling system", says Clifford Rosen, an osteoporosis researcher at the Maine Medical Center Research Institute in Scarborough.

"I think it was very important that Tom came along," says Karsenty. "Novelty comes with the extra price of scrutiny, and the best way to address scrutiny is if your neighbour gets the same results as you."

The link between the skeleton and glucose metabolism has more than just theoretical implications. The model has "great clinical relevance", says Rosen. The most tantalizing possibility in the short term, he says, is that osteocalcin could be used as a way of promoting insulin secretion to treat diabetes. With that in mind, Karsenty spun out a company called Escoublac in late

2007. Head-quartered in Cambridge, Massachusetts, it aims to capitalize on the connections between osteocalcin and bone growth, insulin sensitivity and weight gain.

\section{Thrown a bone}

Despite the therapeutic potential, several pieces of the puzzle are still missing. "I guess if I wasn't in it, I'd be sceptical too," says Clemens. The Karsenty lab has yet to close the leptin loop and identify exactly how bone regulates leptin levels. And so far, neither group has identified an osteocalcin receptor. Both are now examining how osteocalcin might prompt $\beta$-cells to stimulate insulin production.

The main outstanding issue, however, is how closely these studies in mice reflect the physiology of humans. "My personal conviction is that he is right overall, but that it may not be a key component of the human regulatory pathway," says Roland Baron, a bone biologist

\section{CLOSE TO THE BONE}

Bone remodelling includes both building of bone (by cells called osteoblasts) and destruction of bone (by osteoclasts) This dynamic, energy-intensive process seems to interact with metabolism in multiple ways.

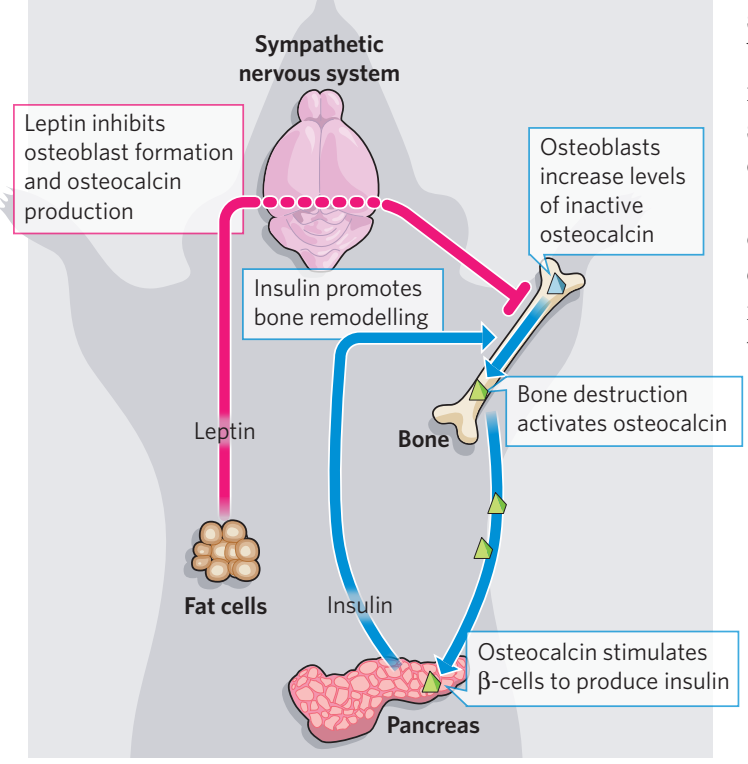

at Harvard School of Dental Medicine in Boston, Massachusetts. "Part of the problem is that [Karsenty] has a tremendous tendency to overstate his findings. And that makes a lot of people uncomfortable." Karsenty says he isn't surprised that his ideas garner criticism. "I will say nothing to them - I will go back to the lab and do more experiments to weaken the resistance."

Rosen says that the work is pushing at the edges of knowledge in valuable ways. "Even if he is $50 \%$ right, he has done a tremendous service to the field." Yet the other thorn in Karsenty's theory, he says, is that women who take medication for osteoporosis to prevent bone resorption - bisphosphonates, for example, or the newer antibody-based drug Prolia (denosumab) that was approved in Europe and the United States in June - don't seem to have significantly altered glucose levels. This could mean that any control bone has on insulin and glucose is minor in humans, says Rosen. Karsenty argues that such drugs decrease osteocalcin levels and insulin secretion resulting in higher glucose levels, but that the change is subtle because they are not potent enough to whack the metabolism far out of balance. Also, he notes, studies in the past few years have reported a link between blood osteocalcin levels and insulin sensitivity in humans ${ }^{6}$.

Although supportive of a link, says Khosla, those observations have largely been made in overweight or obese individuals, who tend to be insulin resistant and have low bone turnover to begin with. "So is it cause and effect, or just two things that happen together but are not causally related?"

Clinical data exist from studies on the osteoporosis drugs, but without a prospective study examining the relationship between osteocalcin and obesity or diabetes in humans, it will be impossible to know for sure. Karsenty is starting to collaborate with clinical researchers to gather such data.

"This has to be proved right or wrong quickly," says Clemens. "There are lots of observations in the basic literature that are not repeated because they are not going to be changing clinical practice." But the implications for current patients make this important, he says. "This won't be left behind."

Alla Katsnelson writes for Nature from New York.

1. Fulzele, K. et al. Cell 142, 309-319 (2010)

2. Ducy, P. et al. Cell 100, 197-207 (2000)

3. Yadav, V. K. et al. Cell 138, 976-989 (2009)

4. Lee, N. K. et al. Cell 130, 456-469 (2007).

5. Ferron, M. et al. Cell 142, 296-308 (2010)

6. Strapazzon, G., De Toni, L. \& Foresta, C. Osteoporos. Int. doi:10.1007/s00198-010-1322-2 (2010). 